\title{
Ownership and utilization of bed nets and reasons for use or non-use of bed nets among community members at risk of malaria along the Thai-Myanmar border
}

\author{
Kasama Pooseesod ${ }^{1,2}$, Daniel M. Parker ${ }^{3}$, Natthani Meemon ${ }^{4}$, Saranath Lawpoolsri ${ }^{1}$, Pratap Singhasivanon ${ }^{1,7}$,
} Jetsumon Sattabongkot ${ }^{5}$, Liwang Cui ${ }^{6}$ and Suparat Phuanukoonnon ${ }^{1,8^{*}}$

\begin{abstract}
Background: With the goal for malaria elimination in Thailand set for 2024, increased coverage and utilization of bed net, especially insecticide-treated net (ITN) or long-lasting insecticidal net (LLIN) is a key strategy. This study aims to provide the necessary information about bed net ownership and utilization among the population at risk of malaria living along the Thai-Myanmar border in Tak province.

Methods: A cross-sectional study was conducted using a mixed-method approach in 331 households from 5 hamlets in the villages of the Thai-Myanmar border. The research tools included a questionnaire, bed net inspection, and semi-structured interviews. Logistic regression was used to explore the sociodemographic factors associated with bed net utilization. The qualitative analysis employed a thematic analysis approach.

Results: This survey found that $98.5 \%$ of households had at least one bed net per household, and $74.3 \%$ had at least one ITN/LLIN. However, only $30.8 \%$ of households reached the standard policy set by the Minister of Public Health of one ITN/LLINs per two persons. Most residents used bed net $(92.1 \%$ used in the previous night and $80.9 \%$ used every day). For those using bed nets, however, $61.9 \%$ used ITNs or LLINs the night before and $53.1 \%$ used them every day. Nonetheless, the usage rates of bed nets (any type) in the previous night among children and pregnant women were high, reaching $95.3 \%$ and $90.0 \%$, respectively. Seven explanatory variables showed statistically significant associations with bed net use every day, including: "not staying overnight in the forest or the field", "sleeping pattern based on gender", "sufficient numbers of bed nets to cover all sleeping spaces", "preference for free bed nets", "age", "gender", and "SES score" showed statistically significant association with bed net use every day. The major reasons for the regular use of bed nets in both household and the forest were to prevent mosquito biting. The reasons for not using bednets in the household were discomfort feelings from heat, perception of unnecessity due to low mosquito density, whereas the reason for not using bed nets in the forest was inconvenience.
\end{abstract}

Conclusion: Despite that overall coverage and usage of bed nets was high, only one third reached the standard level specified by the policy. Overnight in the forest, the dissatisfaction with the quality of free bed nets, insufficient number of bed nets, sleeping alone, male gender, age more than 10 years, low socioeconomic status, discomfort from

*Correspondence: suparat.phu@mahidol.ac.th

1 Department of Tropical Hygiene, Faculty of Tropical Medicine, Mahidol

University, Nakhon Pathom, Thailand

Full list of author information is available at the end of the article

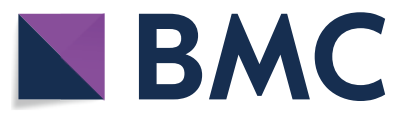

(c) The Author(s) 2021. This article is licensed under a Creative Commons Attribution 4.0 International License, which permits use, sharing, adaptation, distribution and reproduction in any medium or format, as long as you give appropriate credit to the original author(s) and the source, provide a link to the Creative Commons licence, and indicate if changes were made. The images or other third party material in this article are included in the article's Creative Commons licence, unless indicated otherwise in a credit line to the material. If material is not included in the article's Creative Commons licence and your intended use is not permitted by statutory regulation or exceeds the permitted use, you will need to obtain permission directly from the copyright holder. To view a copy of this licence, visit http://creativeco mmons.org/licenses/by/4.0/. The Creative Commons Public Domain Dedication waiver (http://creativecommons.org/publicdomain/ zero/1.0/) applies to the data made available in this article, unless otherwise stated in a credit line to the data. 
heat, perception of no benefits of bed nets due to low mosquito density, and inconvenience were factors influencing bed net use. Maintaining high coverage and utility rate of bed nets should be a priority for the malaria high-risk population.

Keywords: Malaria, Bed net, Long-lasting insecticidal net, Karen ethnic, Forest goers, Thailand

\section{Background}

Based on the 2020 world malaria report, there were 87 countries and areas with ongoing malaria transmission and approximately 229 million malaria cases in 2019 [1].Thailand reported a substantial decline (19\%) in total reported cases between 2018 and 2019 [2]. Malaria in Thailand is patchy in its distribution and can be typified as 'border malaria' and 'forest malaria', with the highest transmission along international borders and in rural forested areas [3]. The western border with Myanmar has had the highest burden of malaria and has been the focus of malaria control programmes for decades [3]. Motivated by the continuous decrease in malaria burden, the Thai government has declared a national malaria elimination plan intending to achieve this by the year 2024 [4].

The major malaria vectors in Thai-Myanmar border include Anopheles minimus sensu lato (s.l.) (40.32\%) and Anopheles maculatus s.l. (21.43\%) [5]. Anopheles minimus was most abundant during the transition from wet to dry season and found more indoor than outdoor. Anopheles maculatus was the most abundant during the wet season in both indoor and outdoor. Both species are typically found in or near hilly, forested regions $[6,7]$. Moreover, Anopheles annularis s.l. and Anopheles barbirostris s.l. were also identified as additional vectors with potential outdoor malaria transmission after the wet season [5]. The potential roles of different anopheline species in human malaria transmission in this region have been the challenge for effective vector control.

To accomplish this elimination goal within the time frame, a key strategy is to increase the bed net coverage and utilization, especially insecticide-treated nets (ITN) or long-lasting insecticidal nets (LLIN). The goal is to achieve $90 \%$ LLIN coverage among populations in high transmission areas (designated as A1 or A2 areas). A1 is village with reported indigenous malaria cases in current financial year and A2 is village without indigenous malaria cases for past 1-3 years [8]. Previous studies among the general population in Thailand and among the populations on the Thai-Myanmar border (Prachuap Khiri Khan Province) identified poor coverage and poor utilization of ITN/LLINs [9, 10]. However, actual coverage and utilization of bed nets as well as factors attributing to bed net use have not been investigated in this study area.
The objective of this study was to assess the ownership, accessibility, and utilization of both treated (ITNs and LLINs) and untreated bed nets in a remaining malaria transmission focus in western Thailand. A cross-sectional study was conducted and questionnaires were used to determine predictors of bed net use. The results are useful for formulating appropriate policies for the control programmes and for the promotion of LLINs and longlasting insecticide-treated hammock net (LLIHN) within the context of populations living along the malarious borders.

\section{Methods \\ Study design}

This is a mixed-methods cross-sectional study that included a questionnaire, an inspection form, and semistructured interviews among selected participants. The household survey was conducted from August to October 2019.

\section{Study site}

A community-based cross-sectional survey was conducted in the areas under the International Center of Excellence for Malaria Research (ICEMR) project in Tha Song Yang District, Tak Province, northwestern Thailand (Fig. 1). Tha Song Yang is situated in the northwestern region of Tak, on the Moei River bank near the Myanmar border. The climate is tropical with an annual average temperature of $26.4{ }^{\circ} \mathrm{C}$. The rainy season is between May and October, with an average yearly rainfall of $1540 \mathrm{~mm}$. The inhabitants of this area are approximately $30 \%$ Thai and $70 \%$ ethnic minorities. Normally, there are two peaks of malaria transmission, one at the beginning of the rainy season (May-August) and the other at the end of the rainy season (October) [11]. Plasmodium vivax and Plasmodium falciparum are the predominant species in this region, although all human malaria parasites, as well as the simian malaria species Plasmodium knowlesi, have been identified in this area [12]. This study was conducted in 5 hamlets including Nong Bua, and Tala Oka (Mae Usu sub-district), Suan Oi, Pha Man, and Ko Ma Nae (Tha Song Yang sub-district).

\section{Study population, sampling and sample size}

For the quantitative component of the study, households residing in the study site were randomly selected from 

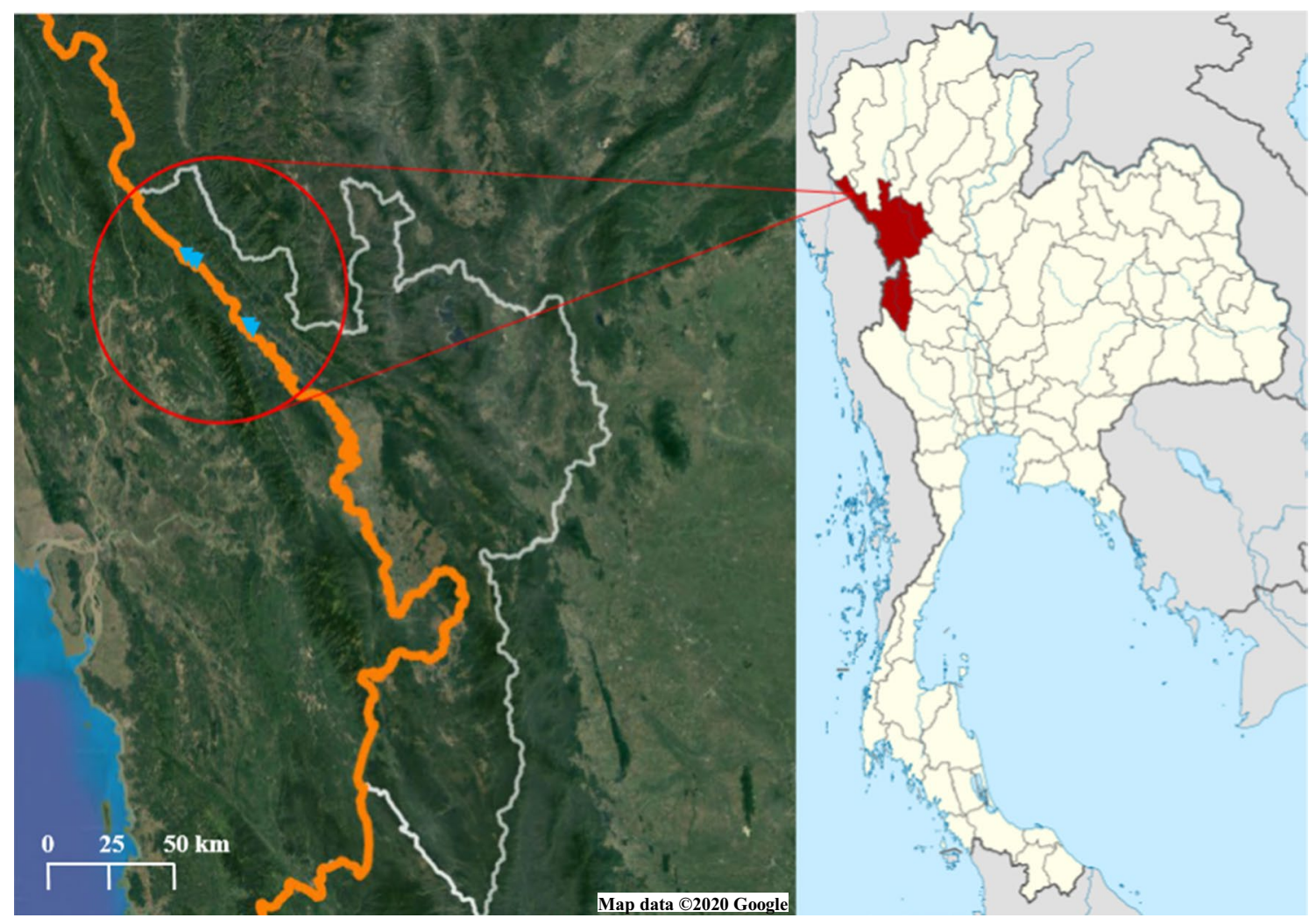

Fig. 1 The study site: Tha Song Yang district, Tak province, Thailand [72, 73]

the study villages. Out of a total of 918 households in the 5 hamlets, 335 were selected by probability proportional to hamlet size, using an online statistical calculator [13]. Most of the household members are the Karen ethnic group [14]. From each household, the questionnaire and inspection form were administered to heads of household or representatives who look after the household. For the qualitative component, 24 potential respondents including 2 community leaders from each sub-district, and 4 heads of household from each hamlet were selected to participate in the semi-structured interview. Purposive sampling was used to recruit participants for this part of the research. The target participation and the potential participants were selected based on geography, age, gender, and reported bed net use (including both those who do and do not report using bed nets). Important community leaders as "gatekeepers" were consulted and engaged in order to identify and invite the participants to join this study. Schematic diagram of the study was illustrated in Fig. 2.

\section{Household surveys}

A community-based survey was conducted to assess the ownership, accessibility, and utilization of both treated
(ITNs and LLINs) and untreated bed nets using a questionnaire, inspection form, and semi-structured interviews. Respondents from 331 households in completed the interviews. In the selected households, face-to-face interviews were conducted with heads of household by a trained study team who speak and understand both Thai and Karen languages.

\section{Data analysis and statistics}

Data were extracted from the survey database and imported into the SPSS program version 22.0 [15] for analysis. Proportions (with 95\% confidence intervals) were used to summarize categorical variables related to ownership, accessibility, and utilization of bed nets. A single variable for socioeconomic status (SES) was generated using factor analysis of mixed data (FAMD), using a combinatoin of continuous and categorical variables (full list in Additional file 1). Logistic regression was then used to explore the sociodemographic factors associated with bed net utilization $(1=$ yes, used bed net; $0=$ no, did not use). The logistic regression model included a random intercept for household to account for confounding and difference in response variation within and between households. Adjusted odds ratios (AOR) (with 


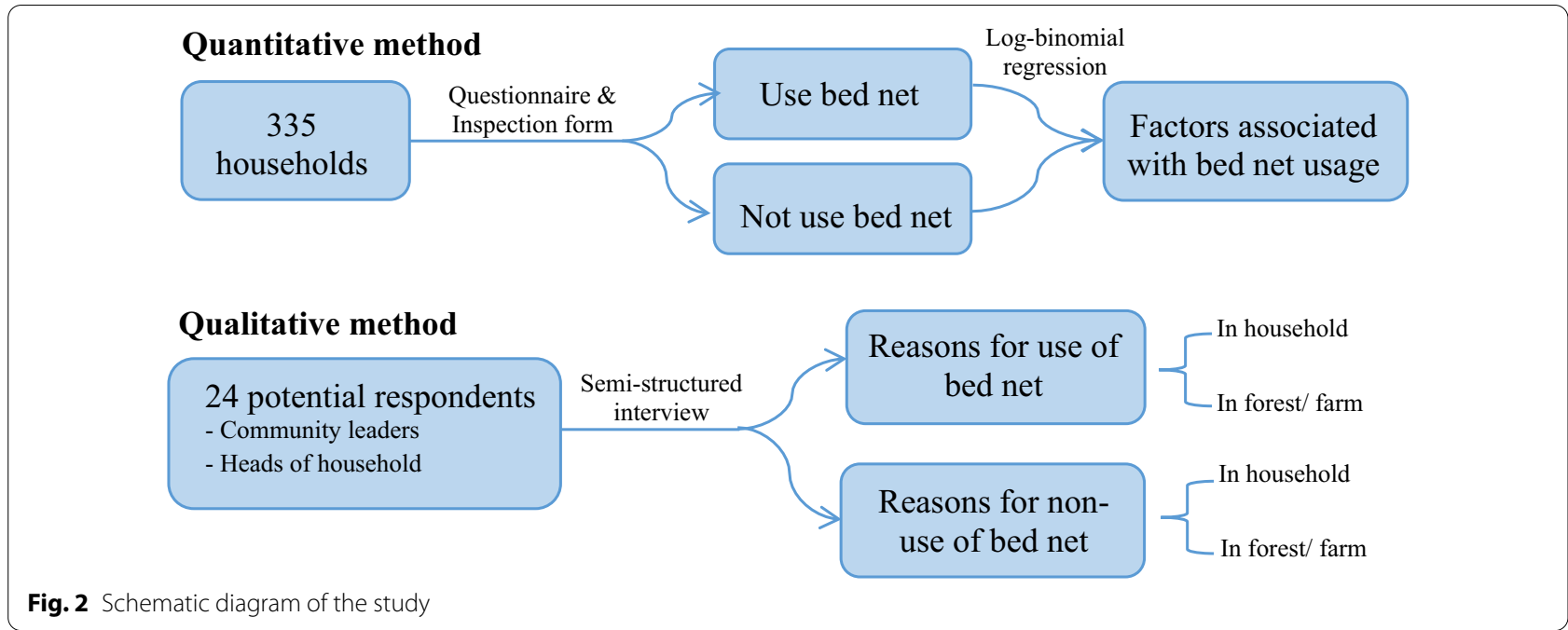

95\% confidence intervals [CIs]) were used to measure the strength of this association.

The qualitative analysis employed a thematic analysis approach [16]. The tentative code categories were given in the conceptual framework. Data were analysed using thematic analysis of content to allow bringing together of similar views from different respondents together.

\section{Results}

\section{Household-level characteristics}

For the 331 households participating in the study, the majority had bamboo/wood walls (94.56\%), bamboo/ wood floors (82.18\%), and terracotta/galvanized iron roofs (71.60\%) (Fig. 3). Among them, 315 (95.17\%) households were from the Karen ethnic minority, and $4.83 \%$ were Thai. Questionnaires were administered to 122 (36.86\%) male and 209 (63.14\%) female household heads. The mean age of the 331 respondents was $43.42( \pm 14.03)$ years; 229 (69.18\%) heads of households were 35 years or older. A large proportion (77.64\%) of the respondents were illiterate. Over half $(54.98 \%)$ of the households had a family income of $\leq 63.80 \mathrm{USD} /$ month (Table 1 ). For the 1,423 household members who reported bed net use, $787(55.31 \%)$ were female (Table 2). The mean age of the household members was $25.68( \pm 20.05)$ years; $620(43.57 \%)$ were $18-59$ years old. For the participating household members, 186 (13.07\%) of whom stayed in the forests or the field.

\section{Bed net ownership, access, and utilization}

Table 3 shows the household-level bed net ownership, access, and utilization. Almost all (98.49\%) households had at least one bed net per household, and $74.32 \%$ had at least one ITNs/LLINs in the household. However, only $30.82 \%$ of all households had sufficient numbers of ITN/ LLINs, as per standard policy i.e., one ITN/LLIN per two persons. Moreover, only $4.30 \%$ of forest goers had owned LLIHNs. Besides, $86.10 \%$ of the households had sufficient numbers of bed net to cover all sleeping spaces. Although $94.17 \%$ of the household members slept in the sleeping spaces with any type of bed nets, $63.46 \%$ slept in sleeping spaces with ITNs or LLINs.

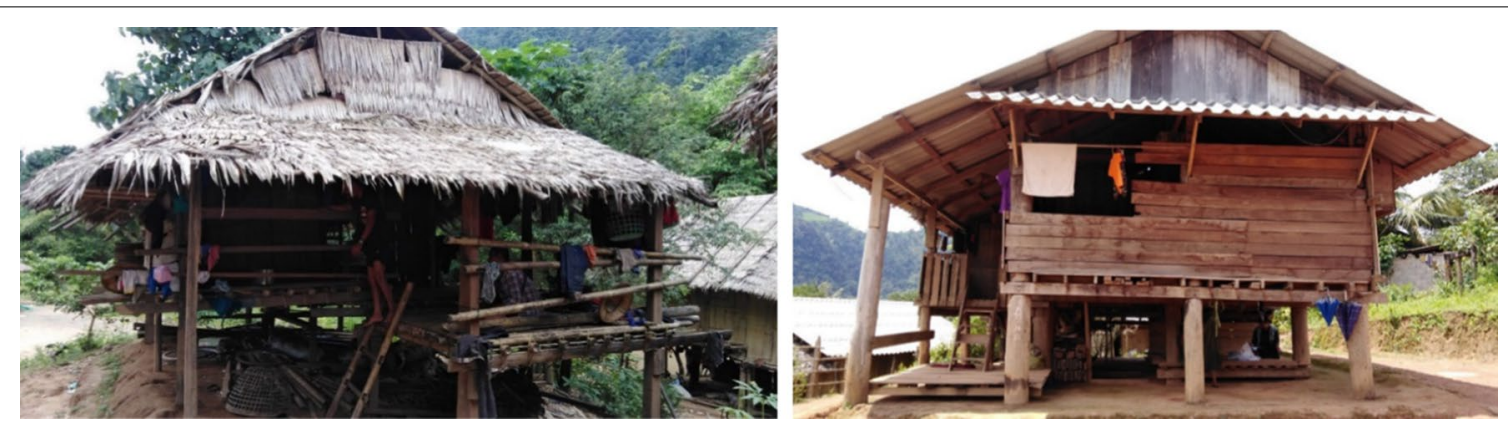

Fig. 3 Housing characteristics of the border population in Tha Song Yang district, Tak province, Thailand 
Table 1 Household and heads of household characteristics of border population in Tha Song Yang district, Tak province, Thailand

\begin{tabular}{|c|c|}
\hline Characteristics & Total $n=331, n(\%)$ \\
\hline \multicolumn{2}{|l|}{ Wall } \\
\hline Bamboo/wood & $313(94.56)$ \\
\hline Concrete & $18(5.44)$ \\
\hline \multicolumn{2}{|l|}{ Floor } \\
\hline Bamboo/wood & $272(82.18)$ \\
\hline Concrete & $59(17.82)$ \\
\hline \multicolumn{2}{|l|}{ Roof } \\
\hline Thatch & $94(28.40)$ \\
\hline Terracotta/galvanized iron & $237(71.60)$ \\
\hline \multicolumn{2}{|l|}{ Sex } \\
\hline Male & $122(36.86)$ \\
\hline Female & $209(63.14)$ \\
\hline \multicolumn{2}{|l|}{ Age (years) } \\
\hline$<35$ & $102(30.82)$ \\
\hline$\geq 35$ & $229(69.18)$ \\
\hline Mean $( \pm S D)$ & $43.42( \pm 14.03)$ \\
\hline \multicolumn{2}{|l|}{ Ethnics } \\
\hline Thai & $16(4.83)$ \\
\hline Karen & $315(95.17)$ \\
\hline \multicolumn{2}{|l|}{ Education level } \\
\hline Illiterate & $257(77.64)$ \\
\hline Literate & $74(22.36)$ \\
\hline \multicolumn{2}{|l|}{ Family income/month (Bath) } \\
\hline$\leq 2000$ & $182(54.98)$ \\
\hline$>2000$ & $149(45.02)$ \\
\hline Mean $( \pm S D)$ & $2630.51( \pm 1321.23)$ \\
\hline
\end{tabular}

Table 2 Household member characteristics of border population in Tha Song Yang district, Tak province, Thailand

\begin{tabular}{lc}
\hline Characteristics & Total $\mathbf{n}=\mathbf{1 4 2 3} \mathbf{n}(\mathbf{\%})$ \\
\hline Sex & \\
Male & $636(44.69)$ \\
Female & $787(55.31)$ \\
Age (years) & \\
$\leq 10$ & $428(30.08)$ \\
$11-17$ & $263(18.48)$ \\
$18-59$ & $620(43.57)$ \\
$\geq 60$ & $112(7.87)$ \\
Mean ( \pm SD) & $25.68( \pm 20.05)$ \\
Household member stay overnight outside in the forest or the field \\
Yes & $186(13.07)$ \\
No & $1237(86.93)$ \\
\hline
\end{tabular}

Table 3 Household ownership access and utilization of bed nets among border population in Tha Song Yang district, Tak province, Thailand

\begin{tabular}{|c|c|}
\hline Characteristics & n (\%) \\
\hline \multicolumn{2}{|l|}{ Household ownership of bed nets } \\
\hline At least one net per household (any type) & $326(98.49)$ \\
\hline At least one ITN/LLIN per household & $246(74.32)$ \\
\hline \multicolumn{2}{|l|}{ Access of bed nets } \\
\hline One net per two people (any type) & $168(50.76)$ \\
\hline One ITN/LLIN per two people & $102(30.82)$ \\
\hline One LLIHN per one people & $8(4.30)$ \\
\hline$\%$ of population with access to any bed net & $1340(94.17)$ \\
\hline$\%$ of population with access to ITN/LLIN & $903(63.46)$ \\
\hline $\begin{array}{l}\% \text { of household have sufficient numbers of bed net to } \\
\text { cover all sleeping spaces }\end{array}$ & $285(86.10)$ \\
\hline \multicolumn{2}{|l|}{ Utilization of bed nets } \\
\hline$\%$ of population slept under any bed net last night & $1311(92.13)$ \\
\hline$\%$ of population slept under an ITN/LLIN last night & $881(61.91)$ \\
\hline$\%$ of forest goers slept under an ITN/LLIN last night & $122(65.59)$ \\
\hline$\%$ of population slept under any bed net every day & $1151(80.89)$ \\
\hline$\%$ of population slept under an ITN/LLIN every day & $756(53.13)$ \\
\hline$\%$ of forest goers slept under an ITN/LLIN every day & $16(8.60)$ \\
\hline $\begin{array}{l}\% \text { of children under } 10 \text { years slept under any bed net last } \\
\text { night }\end{array}$ & $408(95.33)$ \\
\hline $\begin{array}{l}\% \text { of children under } 10 \text { years slept under an ITN/LLIN last } \\
\text { night }\end{array}$ & $255(59.58)$ \\
\hline$\%$ of pregnant women slept under any bed net last night & $10(100.00)$ \\
\hline$\%$ of pregnant women slept under an ITN/LLIN last night & $9(90.00)$ \\
\hline
\end{tabular}

Most of the household members (92.13\%) reported using bed nets in the previous night, and 881 (61.91\%) used ITNs or LLINs (Table 3). Since some respondents did not use bed net every day, especially in the summer season or when staying overnight in the forests or agricultural fields, daily use of bed net was also recorded. While 1,151 (80.89\%) household members reported bed net usage every day, 756 (53.13\%) used ITNs or LLINs. Only $8.60 \%$ of forest goers use ITN/LLIN every day. For children under 10 years $(n=428), 95.33 \%$ slept under a bed net in the previous night, and $59.58 \%$ slept under an ITN/LLIN. Among the pregnant women $(\mathrm{n}=10)$, this was $100 \%$ and $90 \%$, respectively. Out of all 601 inspected bet nets, 109 (18.14\%) had holes (defined as a tear or opening that a finger could fit through). For the age of bed net, $52.08 \%$ of the bed nets were up to 1 year old, $44.59 \%$ were $2-3$ years old, and $3.33 \%$ were more than 3 years old.

The most common pattern of bed net deployment by the household heads included inspecting for holes (86.32\%) and checking for mosquitoes trapped inside (83.06\%). However, only $36.81 \%$ tucked in the nets fully before sleeping, and $28.99 \%$ slept away from the edges 
of the bed. Only 103 (33.55\%) of the household heads deployed the bed nets properly (Table 4).

Sociodemographic factors associated with bed nets usage Univariate logistic regressions were to compare the daily bed nets use across the different variables. Based on the univariate models, strong associations were found between bed net use and stay overnight in the forest or the field [no versus yes: $O R=67.80,95 \%$ $\mathrm{CI}=42.77-107.49 ; \mathrm{p}<0.001]$, sleeping pattern based on same gender [female sleep with female vs male sleep alone: $\mathrm{OR}=15.94,95 \% \mathrm{CI}=7.00-36.27 ; \mathrm{p}<0.001]$, sufficient numbers of bed net to cover all sleeping spaces [yes versus no: $\mathrm{OR}=6.03,95 \% \mathrm{CI}=4.28-8.48$; $\mathrm{p}<0.001$ ], preference for free bed net [yes or indifferent vs not like: $\mathrm{OR}=5.50,95 \% \mathrm{CI}=3.36-9.02 ; \mathrm{p}<0.001]$, and age $[18-59 v s \leq 10$ : $\mathrm{OR}=0.08,95 \% \mathrm{CI}=0.04-0.14$; $\mathrm{p}<0.001]$. Additionally, a weak association was found between daily bed net use and marital status [others vs married: $\mathrm{OR}=3.62,95 \% \mathrm{CI}=2.70-4.87 ; \mathrm{p}<0.001]$, gender [female $v s$ male: $\mathrm{OR}=3.20,95 \% \mathrm{CI}=2.39-4.28$; $\mathrm{p}=<0.001]$, sleeping pattern based on younger age ( $\leq 10$ year) [sleep with child $v s$ not sleep with child: $\mathrm{OR}=2.65,95 \% \mathrm{CI}=2.00-3.52 ; \mathrm{p}<0.001]$. However, SES score was not significantly associated with bed net use.

In the final multivariable logistic regression, seven explanatory variables including: "not staying overnight in the forest or the field", "sleeping pattern based on gender", "sufficient numbers of bed net to cover all sleeping spaces", "preference for free bed net", "age”, "gender", and "SES score" showed a significant association with daily bed net use (Table 5).

Table 4 Pattern of deployment of bed net by head of household owning and using bed net $(n=307)$

\begin{tabular}{lrr}
\hline & $\mathbf{n}$ & $\%$ \\
\hline Pattern of deployment of bed nets & \\
Tucking in fully during sleeping & 113 & 36.81 \\
Inspecting for holes & 265 & 86.32 \\
Checking for mosquitos trapped inside & 255 & 83.06 \\
Sleeping away from the edge of the bed & 89 & 28.99 \\
Head of household deployed bed net properly & & \\
Yes & 103 & 33.55 \\
No & 204 & 66.45 \\
Total & 307 & 100.00 \\
\hline
\end{tabular}

${ }^{a}$ Multiple responds allowed
Reasons for use or non-use of bed nets in the household and the forest

A total of 22 respondents were interviewed by semistructured interview. The results organized into key themes that emerged from the discussions.

\section{Reasons for use of bed nets in the household}

Most participants reported using bed nets to prevent mosquito bites and malaria infection. Most of them remembered using bed nets from a very young age, whereas some of them started to use bed nets when they had children or grandchildren. Bed net use had become a habit for them and they could not sleep without it.

"I use bed nets to protect myself against mosquito bites. I have used bed nets since I was born and becoming my habit I cannot sleep without the net" (Female villager, Mae Usu subdistrict).

Additionally, free bed net distribution campaigns were mentioned as a factor that supported them to start and continue using bed nets in the last 20-30 years.

"After I immigrated to Thailand 20 years ago, I started to use bed nets and they were free bed nets distributed from health providers. The net is good and prevents mosquitoes from biting and not getting sick with malaria." (Female villager, Tha Song Yang subdistrict).

\section{Bed net use in farms and forests}

Forest goers in this area had not used any kinds of bed net while they were in the forest. Most forest goers used bed net only in the subsistence farm huts, their temporary residence nearby the forest. Both forest goers and farmers, if they had sufficient bed nets for use in their households, they would take the old bed nets for use in the subsistence farm huts. If they had limited bed nets, they would carry bed net to the subsistence farm hut and carry it back home, especially when children accompanied them to the farms. However, many of them used bed nets in a subsistence farm hut only in the rainy season when mosquitoes are abundant.

"When children accompanied us to the farm plots or forest, we always carried bed net to use in the farm hut. Our children need to get protection from mosquito bites." (Female villager, Mae Usu subdistrict).

"I sleep under bed net every day. And even when I was going to overnight in the farm, I took an old bed net to use in the farm hut." (Male villager, Tha Song Yang subdistrict).

\section{Reasons for non-use of bed nets in the household}

The major reason that participants reported for not using bed nets every day at home was discomfort from 
Table 5 Variables related to use of bed nets every day among respondents living in households owning at least one net ( $\mathrm{n}=1401$ )

\begin{tabular}{|c|c|c|c|c|c|}
\hline & \multirow[t]{2}{*}{ n (\%) } & \multicolumn{2}{|l|}{ Univariate analysis } & \multicolumn{2}{|c|}{ Multivariable analysis } \\
\hline & & OR $(95 \% \mathrm{Cl})$ & p-value & OR $(95 \% \mathrm{Cl})$ & p-value \\
\hline \multicolumn{6}{|l|}{ Age of household member (years) } \\
\hline$\leq 10(n=418)$ & $405(96.89)$ & 1 & - & - & - \\
\hline $11-17(n=259)$ & $220(84.94)$ & $0.17(0.09-0.33)$ & $<0.001^{* *}$ & $0.17(0.07-0.43)$ & $<0.001^{* *}$ \\
\hline $18-59(n=613)$ & $442(72.10)$ & $0.08(0.04-0.14)$ & $<0.001^{* *}$ & $0.13(0.04-.36)$ & $<0.001^{* *}$ \\
\hline$\geq 60(n=111)$ & $84(75.68)$ & $0.09(0.04-0.18)$ & $<0.001^{* *}$ & $0.24(0.07-0.85)$ & $0.027^{*}$ \\
\hline \multicolumn{6}{|l|}{ Gender of household member } \\
\hline Male $(n=625)$ & $456(72.96)$ & 1 & - & - & - \\
\hline Female $(n=776)$ & $695(89.56)$ & $3.20(2.39-4.28)$ & $<0.001^{* *}$ & $2.44(1.41-4.22)$ & $0.001^{*}$ \\
\hline \multicolumn{6}{|l|}{ Marital status of household member } \\
\hline Married $(n=610)$ & $439(71.97)$ & 1 & - & - & - \\
\hline Others $(n=791)$ & $712(90.01)$ & $3.62(2.70-4.87)$ & $<0.001^{* *}$ & $0.66(0.29-1.47)$ & 0.304 \\
\hline \multicolumn{6}{|l|}{ Household member stay overnight outside in the forest or the field } \\
\hline Yes $(n=184)$ & $27(14.67)$ & 1 & - & - & - \\
\hline No $(n=1,217)$ & $1124(92.36)$ & $67.80(42.77-107.49)$ & $<0.001^{* *}$ & $96.25(52.17-177.58)$ & $<0.001^{* *}$ \\
\hline \multicolumn{6}{|l|}{ Head of household prefer to use free bed net } \\
\hline Not prefer $(n=75)$ & $34(45.33)$ & 1 & - & - & - \\
\hline Yes or indifferent $(n=1,326)$ & $1117(84.24)$ & $5.50(3.36-9.02)$ & $<0.001^{* *}$ & $13.42(6.47-27.85)$ & $<0.001^{* *}$ \\
\hline \multicolumn{6}{|l|}{ Sufficient numbers of bed net to cover all sleeping spaces in household } \\
\hline No $(n=190)$ & $104(54.74)$ & 1 & - & - & - \\
\hline Yes $(n=1,211)$ & $1047(86.46)$ & $6.03(4.28-8.48)$ & $<0.001^{* *}$ & $7.22(4.29-12.14)$ & $<0.001^{* *}$ \\
\hline \multicolumn{6}{|l|}{ Sleeping pattern based on gender } \\
\hline Male sleep alone $(n=98)$ & $61(62.24)$ & 1 & - & - & - \\
\hline Female sleep alone $(n=75)$ & $63(84.00)$ & $3.34(1.58-7.06)$ & $0.002^{*}$ & $1.00(0.33-3.06)$ & 0.998 \\
\hline Male sleep with female (spouse) $(n=162)$ & $104(64.20)$ & $1.18(0.70-2.00)$ & 0.536 & $1.53(0.57-4.10)$ & 0.399 \\
\hline Male sleep with male ( $\geq 2$ person/sleeping space) $(n=57)$ & $43(75.44)$ & $2.17(1.04-4.55)$ & $0.040^{*}$ & $1.41(0.43-4.59)$ & 0.568 \\
\hline $\begin{array}{l}\text { Male sleep with female (not spouse) }(\geq 2 \text { person/sleeping space) } \\
\quad(n=808)\end{array}$ & $687(85.02)$ & $4.00(2.51-6.35)$ & $<0.001^{* *}$ & $2.88(1.13-7.37)$ & $0.027^{*}$ \\
\hline Female sleep with female ( $\geq 2$ person/sleeping space) $(n=201)$ & $193(96.02)$ & $15.94(7.00-36.27)$ & $<0.001^{* *}$ & $4.42(1.27-15.47)$ & $0.020^{*}$ \\
\hline \multicolumn{6}{|l|}{ Sleeping pattern based on age ( $\leq 10$ year) } \\
\hline Not sleep with child $\leq 10$ year $(n=533)$ & $394(73.92)$ & 1 & - & - & - \\
\hline Sleep with child $\leq 10$ year $(n=868)$ & $757(87.21)$ & $2.65(2.00-3.52)$ & $<0.001^{* *}$ & $0.72(0.35-1.47)$ & 0.370 \\
\hline \multicolumn{6}{|l|}{ SES score } \\
\hline $\bar{x} \pm S D$ & $0.07 \pm 0.11$ & $2.16(0.53-8.76)$ & 0.282 & $13.93(2.07-93.88)$ & $0.007^{*}$ \\
\hline
\end{tabular}

${ }^{*}$ p-value $<0.05,{ }^{* *} p$-value $<0.001$

the heat and perception of unnecessity due to low mosquito density, especially in the hot season.

"I am still afraid of malaria, but I could not sleep it was too hot to sleep under the nets in the summer" (Female villager, Tha Song Yang subdistrict).

The second most commonly reported reason was feeling complacent and not wanting to expend the effort needed to set up and use nets in the evenings, especially for adolescents who did not share sleeping space with their parents.

"My son and daughter rarely use bed net because they are so lazy to hang the bed net up. I sometimes have to hang it for them and tell them to sleep under bed net." (Female villager, Mae Usu subdistrict).

Other reasons mentioned for not using a net at home included inadequate number of bed nets, being not habitual of sleeping under the bed nets, use of alternative mosquito control methods, too small size of the free bed net, rough texture of material of free bet nets, strong smell of insecticide, and inadequate space to hang a bed net.

\section{Reasons for non-use of bed nets in the forest or farms}

The major reason for the non-use of bed nets in the forest was inconvenient to carry the bed nets and prioritized 
other essential items. Even in the subsistence farm hut, some felt lazy to unpack and hang the bed nets up before sleeping in. Although someone received an LLIHN to use in the forest, they tried to use the hammocks in the forest, they found problems from using the net.

"Going to forest, we travel light with a few necessary items so we can carry lots forest products when we return. It is inconvenient to bring the net to the forest, it takes space to carry. It also can get entangled with other items, obstructed hunting and gatering process, in particular to hunting we need to be quick to shoot the targets. Sometime at night, animals such as elephants approach our sleeping area, we have to run away, we have no time to pack the net." (Male villager, Tha Song Yang subdistrict).

The second reason was the inadequate number of the nets for use when visiting the forest. Other reasons for not using a net in the forest included being not habitual of sleeping under the bed nets, discomfort from heat, and use of alternative mosquito control methods.

\section{Discussion}

This is the first cross-sectional study assessing the ownership and utilization of bed nets among the border population in Tha Song Yang District of western Thailand. From the result, bed nets are widely available in these localities, a result of efforts by the Department of Disease Control and other partners (such as non-governmental organizations) that have distributed free LLINs from the Global Fund [9]. However, some households did not have ITNs/LLINs. These results are consistent with the finding among the general population in Thailand and among the populations on the Thai-Myanmar border (Prachuap Khiri Khan Province) showed high ownership of bed nets, but poor coverage of ITNs $[9,10]$. Similar studies in Myanmar showed high coverage of bed nets with coverage of ITN/LLIN among households of migrant population $[17,18]$.

This study showed only one third households had sufficient numbers of ITN/LLIN for one ITN/ LLINs per two persons. This was below the standard level of WHO recomendations for universal coverage of ITN/ LLINs [19]. This insufficiency might be due to the insufficient number of LLINs obtained from the MOPH and operational challenges of the LLIN distribution system, as some studies identified operational barriers to LLIN distribution [20,21]. This study suggests that the performance of LLIN distribution system and the operational challenges of LLIN distribution in Thailand should be evaluated, and the insufficiency of ITN/LLIN is also a concern and should be used as an indicator for the LLIN distribution programme. A study in Mozambique reported that the access indicators of LLINs were high when the bed net distribution campaigns used a novel distribution model and used LLINs designated for each sleeping space [22].

Globally, malaria-related morbidity and mortality are highest in children and pregnant women [23]. Overall bed net usage among adults in this study was lower than those among children and pregnant women. This was mainly due to the high focus on vulnerable groups. Sleeping with a female household member (i.e. father with daughter, mother with son) was also associated with higher bed net usage when compared with males sleeping alone. However, approximately a half of the children in this study used ITNs/LLINs, consistent with the previous findings in Myanmar and some countries in Africa which showed that the utilization of ITN remained moderate or low among children. [17, 18, 24, 25], One of the reasons for low untilization were insufficiency of ITN/ LLIN among migrant of Myanmar [17, 18]. Conversely, the factors significantly associated with children ITNs utilization in Ethiopia and Ghana were caretaker's age being $<30$ years, small family size ( $\leq 5$ members), and more of sleeping spaces $(\geq 2)[24,25]$. Pregnant women, particularly primigravidas with malaria, have a high risk of severe malaria and low birth weights $[26,27]$. This study showed a high rate of ITN/LLIN use among pregnant women. However, in many parts of the world, ITN usage remained moderate or low among pregnant women [18, 28-31].

Most bed nets effectively protect against mosquitoes within three years of WHO's recommended use [32]. Nonetheless, to increase the effective protection, bed nets need regular re-impregnation with insecticide and get holes repaired. Nets may be less effective if not deployed properly such as tucking in properly or being lifted multiple times over the course the night [33]. Despite providing health education during LLIN distribution (head of the Vector-Borne Disease Unit 2.3.6. personal communication), most people still did not deploy bed nets properly and some did not use the nets every day. This finding suggests the need for new motivational approaches or tools to encourage proper use of nets and using bed nets every day.

One of major factors in bed net use was net manufacturing materials and size affecting net utilization among households with more family members [10]. Many other studies also reported under usage of nets due to the insufficient nets for all household members $[10,17,34$, 35]. Household socio-economic status has likewise been shown to influence bed net access and is the strongest determinant of net use [30, 36-43]. This study showed that wealthier families were significantly more likely to use bed nets every day than the poorer families, likely because of the ability to purchase bed nets. 
Consistent with previous studies [33, 44-46], the perceived role of bed nets as a means to protect against mosquitoes and malaria was a significant predictor of seasonal bed net use. In this study, participants reported higher bed net usage during the rainy season. Aside from malaria prevention, the main reason for sleeping under nets was because they provided comfortable sleep and protected against biting insects (including mosquitoes).

Other reasons for the not using bed nets were in line with other research $[10,18,47-52]$ suggesting that discomfort from heat and low mosquito density are common reasons for non-adherence. Complacence, the inadequate number of of bed net, rough materials of bed net, not having a routine of bed net use, use of alternative mosquito control methods, and too small size of free bed nets were the other most commonly cited reasons for not using a net at home. Some studies have shown that bed nets decrease airflow, making it feel hot and stifling under a net $[53,54]$. In seasons of low and/or variable mosquito nuisance, education will need to emphasize that the risk of malaria is not necessarily diminished when mosquito numbers are perceived to be low [55].

Work and evening activities in the forest affected where community members choose to sleep on any particular evening. Together with the previous findings in Prachuab Khiri Khan province [10] and Southern Thailand [56], sleeping elsewhere especially at temporary shelters in the plantation was main non-use reason. The reasons for not using bed net in the forest were also consistent with studies in the Greater Mekong Subregion [57-59]. Some forest-goers described the inconvenience of carrying bed nets, while others found inadequacy of nets for use when visiting the forest. This study suggests that the campaign of LLIHN distribution appeared not effective for malaria prevention among forest goers. The advantages of these nets might be not adequately promoted as well as other strategies to address outdoor transmission may need to be considered among this population [60-62].

Previously reported limited effectiveness of bed nets in Southeast Asia may have resulted from the vector feeding behaviour and human activities that in some circumstances increase human-vector contacts [63, 64]. Some main malaria vectors, such as An. minimus, An. maculatus and Anopheles dirus feed outdoors at dusk between 6 and 7 p.m. when people are not in bed [63, 65]. Also, forest goers exhibit behaviors (hunting, gathering or fishing) that reduce the protection of bed nets (ITNs) at peak biting times [66, 67]. Nevertheless, given the broader impacts of ITNs for preventing all vector-borne diseases, such as malaria [68, 69], Japanese encephalitis [70], and leishmaniasis [71], proper net distribution and encouraged use still need to be strengthened, especially for the high-risk populations.
For the study limitations, this study was conducted in Thasongyang district; therefore, the results could represent population living along Thai-Myanmar border and Karen ethnic groups, but not represent Karen groups from other areas in which lifestyle, culture, or health operation were different. Due to the limitation of budget and time, this study was conducted in one season (rainy season with more abundant of mosquitoes) which may result in high rate of bed net use in the previous night. Nonetheless, adding question of everyday use of bed net, should reduce the bias from season differences. Recall bias may exist, especially with the questions regarding actual age of bed nets; some respondents could not recall the distribution date or purchase date. This study did not assess the impact of bed nets on malarial infections, therefore; furture research needs to address this aspect.

\section{Conclusions}

This study showed that high overall coverage and usage of bed nets in the study area; however, only one third reached the standard level specified by the policy. Overnighting in the forest or the farm plots, the dissatisfaction with the quality of free bed nets, insufficient number of bed nets, sleeping alone, male gender, age more than 10 years, low socioeconomic status, discomfort from heat, perception of no benefits of bed nets due to low mosquito density, and inconvenience were factors influencing bed net use. Moreover, only one third of heads of households deployed bed net properly. Further health promotion programmes should ensure to maintain high coverage and utility rate of bed nets and further studies should prioritize the investigation of new preventive tools for effective outdoor protection in particualt ot the forest goers.

\section{Abbreviations \\ ITN: Insecticide-treated net; LLIN: Long-lasting insecticidal net; LLIHN: Long- lasting insecticide-treated hammock net; ICEMR: International Center of Excel-} lence for Malaria Research; PR: Prevalence ratio; Cl: Confidence interval.

\section{Supplementary Information}

The online version contains supplementary material available at https://doi. org/10.1186/s12936-021-03837-5.

Additional file 1: The list of socioeconomic status (SES) variables.

Acknowledgements

We thank all participating community member, community leaders, and health staffs for their dedicated study participation.

Authors' contributions

KP, SP, and PS conceived and designed the study. KP and SP collected data and conducted interviews. KP and SL participated in sample processing and data analysis. KP, DMP, NM, SL, PS, JS, LC, and SP participated in data analysis, 
interpretation, and drafting the manuscript. All authors have read and approved the final manuscript.

\section{Funding}

This study was funded by the National Institute of Allergy and Infectious Diseases, National Institutes of Health (U19 Al089672) and Faculty of Tropical Medicine, Mahidol University, Thailand.

\section{Availability of data and materials}

The datasets used and/or analysed during the current study are not provided.

\section{Declarations}

\section{Ethics approval and consent to participate}

The heads of households or adult representatives were approached for consenting before recruitment. A written informed consent was obtained as permission to conduct data collection. The study was approved by the Ethics Committee of the Faculty of Tropical Medicine, Mahidol University, Thailand (Ethical number MUTM 2019-056-01).

\section{Consent for publication}

All authors gave the consent for this publication.

\section{Competing interests}

The authors declare that they have no competing interests.

\section{Author details}

${ }^{1}$ Department of Tropical Hygiene, Faculty of Tropical Medicine, Mahidol University, Nakhon Pathom, Thailand. ${ }^{2}$ Faculty of Public Health, Thammasat University, Bangkok, Thailand. ${ }^{3}$ Department of Population Health \& Disease Prevention, Program in Public Health Susan and Henry Samueli College of Health Sciences, University of California, Irvine, USA. ${ }^{4}$ Department of Society and Health, Faculty of Social Sciences and Humanities, Mahidol University, Nakhon Pathom, Thailand. ${ }^{5}$ Mahidol Vivax Research Unit, Faculty of Tropical Medicine, Mahidol University, Nakhon Pathom, Thailand. ${ }^{6}$ Department of Internal Medicine, Morsani College of Medicine, University of South Florida, Tampa, FL, USA. ${ }^{7}$ Faculty of Tropical Medicine, SEAMEO TROPMED Regional Centre for Tropical Medicine, Mahidol University, Nakhon Pathom, Thailand. ${ }^{8}$ Department of Social and Environmental Medicine, Faculty of Tropical Medicine, Mahidol University, Nakhon Pathom, Thailand.

\section{Received: 8 April 2021 Accepted: 27 June 2021}

Published online: 06 July 2021

\section{References}

1. WHO. World malaria report 2020. World Health Organization, Geneva, 2020.

2. Division of Vector-Borne Diseases, Department of Disease Control, Ministry of Public Health, Thailand. Database of Thailand malaria elimination program. http://malaria.ddc.moph.go.th/malariaR10/index_v2.php. Accessed 5 Mar 2021.

3. Division of Vector-Borne Diseases, Department of Disease Control, Ministry of Public Health, Thailand. Annual Report Malaria situation in Thailand 2018. Ministry of Public Health, Nonthaburi, 2018.

4. Division of Vector-Borne Disease, Department of Disease Control, Ministry of Public Health, Thailand. National Strategic Plan for Malaria Control and Elimination in Thailand 2017-2026. Ministry of Public Health, Nonthaburi, 2017.

5. Sriwichai P, Samung Y, Sumruayphol S, Kiattibutr K, Kumpitak C, Payakkapol A, et al. Natural human Plasmodium infections in major Anopheles mosquitoes in western Thailand. Parasit Vectors. 2016;9:17.

6. Oo TT, Storch V, Becker N. Review of the anopheline mosquitoes of Myanmar. JVector Ecol. 2004;29:21-40.

7. Sinka ME, Bangs MJ, Manguin S, Chareonviriyaphap T, Patil AP, Temperley WH, et al. The dominant Anopheles vectors of human malaria in the AsiaPacific region: occurrence data, distribution maps and bionomic précis. Parasit Vectors. 2011;4:89.
8. Division of Vector-Borne Diseases, Department of Disease Control, Ministry of Public Health, Thailand. A malaria elimination operational manual for Communicable Disease Control Unit and Surveillance and Rapid Response Team. Ministry of Public Health, Nonthaburi, 2019.

9. President's malaria initiative Thailand, Lao PDR, and regional. Malaria Operational Plan FY 2019. 2019.

10. Kitidamrongsuk P, Jareinpituk S, Pattanasin S, Viwatwongkasem C, Soontornpipit P, Silabutra J, et al. Determinants of impregnated net ownership and utilization in rural community on the Thai-Myanmar border in Prachuab Khiri Khan, Thailand. Procedia Comput Sci. 2016;86:224-7.

11. Cui L, Mascorro CN, Fan Q, Rzomp KA, Khuntirat B, Zhou G, et al. Genetic diversity and multiple infections of Plasmodium vivax malaria in Western Thailand. Am J Trop Med Hyg. 2003;68:613-9.

12. Baum E, Sattabongkot J, Sirichaisinthop J, Kiattibutr K, Jain A, Taghavian $\mathrm{O}$, et al. Common asymptomatic and submicroscopic malaria infections in Western Thailand revealed in longitudinal molecular and serological studies: a challenge to malaria elimination. Malar J. 2016;15:333.

13. Dhand NK, Khatkar MS. Statulator: An online statistical calculator. Sample Size Calculator for Comparing Two Independent Proportions. http://statu lator.com/SampleSize/ss2P.html. Accessed 20 Apr 2019.

14. Lawpoolsri S, Sattabongkot J, Sirichaisinthop J, Cui L, Kiattibutr K, Rachaphaew N, et al. Epidemiological profiles of recurrent malaria episodes in an endemic area along the Thailand-Myanmar border: a prospective cohort study. Malar J. 2019;18:124.

15. IBM Corp. Released. IBM SPSS Statistics for Windows, Version 22.0. Armonk, NY: IBM Corp. 2013.

16. Braun V, Clarke V. Using thematic analysis in psychology. Qual Res Psychol. 2006;3:77-101.

17. Maung TM, Tripathy JP, Oo T, Oo SM, Soe TN, Thi A, et al. Household ownership and utilization of insecticide-treated nets under the Regional Artemisinin Resistance Initiative in Myanmar. Trop Med Health. 2018;46:27.

18. Linn SY, Maung TM, Tripathy JP, Shewade HD, Oo SM, Linn Z, et al. Barriers in distribution, ownership and utilization of insecticide-treated mosquito nets among migrant population in Myanmar, 2016: a mixed methods study. Malar J. 2019;18:172.

19. MEASURE Evaluation, MEASURE DHS, President's Malaria Initiative, Roll Back Malaria Partnership, UNICEF, World Health Organization. Household Survey Indicators for Malaria Control. 2013.

20. Theiss-Nyland K, Kone D, Karema C, Ejersa W, Webster J, Lines J. The relative roles of ANC and EPI in the continuous distribution of LLINs: a qualitative study in four countries. Health Policy Plan. 2017;32:467-75.

21. Webster J, Kayentao K, Diarra S, Diawara SI, Haiballa AA, Doumbo OK, Hill $J A$. qualitative health systems effectiveness analysis of the prevention of malaria in pregnancy with intermittent preventive treatment and insecticide treated nets in Mali. PLoS ONE. 2013;8:e65437.

22. Plucinski MM, Chicuecue S, Macete E, Colborn J, Yoon SS, Kachur SP, et al. Evaluation of a universal coverage bed net distribution campaign in four districts in Sofala Province, Mozambique. Malar J. 2014;13:427.

23. Schantz-Dunn J, Nour NM. Malaria and pregnancy: a global health perspective. Rev Obstet Gynecol. 2009;2:186-92.

24. Diema KK, Japiong M, Dodam KK, Afaya A, Salia SM, Kombat JM. Utilization of insecticide treated bed nets (ITNs) among caregivers of children under five years in the Ho Municipality. Interdiscip Perspect Infect Dis. 2019;2019:3693450.

25. Admasie A, Zemba A, Paulos W. Insecticide-treated nets utilization and associated factors among under-5 years old children in Mirab-Abaya District, Gamo-Gofa Zone, Ethiopia. Front Public Health. 2018;6:7.

26. Tsuang A, Lines J, Hanson K. Which family members use the best nets? An analysis of the condition of mosquito nets and their distribution within households in Tanzania. Malar J. 2010;9:211.

27. Desai MR, Kuile FO, Nosten F, McGready R, Asamoa K, Brabin BJ, et al. Epidemiology and burden of malaria in pregnancy. Lancet Infect Dis. 2007;7:93-104.

28. Inungu JN, Ankiba N, Minelli M, Mumford V, Bolekela D, Mukoso B, et al. Use of insecticide-treated mosquito net among pregnant women and guardians of children under five in the Democratic Republic of the Congo. Malar Res Treat. 2017;2017:5923696.

29. Doda Z, Solomon T, Loha E, Gari T, Lindtjørn B. A qualitative study of use of long-lasting insecticidal nets (LLINs) for intended and unintended purposes in Adami Tullu, East Shewa Zone, Ethiopia. Malar J. 2018;17:69. 
30. Xu JW, Liao YM, Liu H, Nie RH, Havumaki J. Use of bed nets and factors that influence bed net use among Jinuo Ethnic Minority in southern China. PLoS ONE. 2014;9:e103780.

31. Fuge TG, Ayanto SY, Gurmamo FL. Assessment of knowledge, attitude and practice about malaria and ITNs utilization among pregnant women in Shashogo District, Southern Ethiopia. Malar J. 2015;14:235.

32. WHO. Guidelines for monitoring the durability of long-lasting insecticidal mosquito nets under operational conditions. World Health Organization, Geneva. 2011.

33. Msellemu D, Shemdoe A, Makungu C, Mlacha Y, Kannady K, Dongus S, et al. The underlying reasons for very high levels of bed net use, and higher malaria infection prevalence among bed net users than nonusers in the Tanzanian city of Dar es Salaam: a qualitative study. Malar J. 2017;16:423.

34. Min KT, Maung TM, Oo MM, Oo T, Lin Z, Thi A, et al. Utilization of insecticide-treated bed nets and care-seeking for fever and its associated socio-demographic and geographical factors among under-five children in different regions: evidence from the Myanmar Demographic and Health Survey, 2015-2016. Malar J. 2020;19:7.

35. Raghavendra K, Chourasia MK, Swain DK, Bhatt RM, Uragayala S, Dutta GDP, et al. Monitoring of long-lasting insecticidal nets (LLINs) coverage versus utilization: a community-based survey in malaria endemic villages of Central India. Malar J. 2017;16:467.

36. Sangare LR, Weiss NS, Brentlinger PE, Richardson BA, Staedke SG, Kiwuwa MS, et al. Determinants of use of insecticide treated nets for the prevention of malaria in pregnancy in Jinja, Uganda. PLOS ONE. 2012;7:e39712.

37. Hetzel MW, Gideon G, Lote N, Makita L, Siba PM. Ownership and usage of mosquito nets after four years of large-scale free distribution in Papua New Guinea. Malar J. 2012;11:192.

38. Bowen HL. Impact of a mass media campaign on bed net use in Cameroon. Malar J. 2013;12:36.

39. Koenker H, Kilian A. Recalculating the net use gap: a multi-country comparison of ITN use versus ITN access. PLoS ONE. 2014;9:e97496.

40. Kanyangarara M, Hamapumbu H, Mamini E, Lupiya J, Stevenson JC, Mharakurwa S, et al. Malaria knowledge and bed net use in three transmission settings in southern Africa. Malar J. 2018;17:41.

41. Singh M, Brown G, Rogerson SJ. Ownership and use of insecticidetreated nets during pregnancy in sub-Saharan Africa: a review. Malar J. 2013;12:268.

42. Sena LD, Deressa WA, Ali AA. Predictors of long-lasting insecticidetreated bed net ownership and utilization: evidence from communitybased cross-sectional comparative study, Southwest Ethiopia. Malar J. 2013;12:406.

43. Eteng M, Mitchell S, Garba L, Ana O, Liman M, Cockcroft A, et al. Socioeconomic determinants of ownership and use of treated bed nets in Nigeria: results from a cross-sectional study in Cross River and Bauchi States in 2011. Malar J. 2014;13:316.

44. Koenker H, Munoz B, Boulay M, Mkocha H, Levens J, West SK, et al. Trends in weekly reported net use by children during and after rainy season in central Tanzania. Malar J. 2012;11:218.

45. Vanden EJ, Thwing J, Wolkon A, Kulkarni MA, Manya A, Erskine M, et al. Assessing bed net use and non-use after long-lasting insecticidal net distribution: a simple framework to guide programmatic strategies; insecticide-treated bed nets and curtains for preventing malaria. Malar J. 2010;9:133.

46. Atieli HE, Zhou G, Afrane Y, Lee M-C, Mwanzo I, Githeko AK, et al. Insecticide-treated net (ITN) ownership, usage, and malaria transmission in the highlands of western Kenya. Parasit Vectors. 2011;4:113.

47. Adaji J, Gabriel OE. Access and usage of long-lasting insecticidal nets (LLIN) in rural Communities of Benue State, Nigeria. Health Sci J. 2019;3:1-4.

48. Adebayo AM, Akinyemi OO, Cadmus EO. Ownership and of mosquito nets among caregivers of under-five children and pregnant women in a rural community in Southwest Nigeria. J Prev Med Hyg. 2014;55:58-64.

49. Solomon T, Loha E, Deressa W, Gari T, Overgaard HJ, Lindtjørn B. Low use of long-lasting insecticidal nets for malaria prevention in south-central Ethiopia: a community-based cohort study. PLoS ONE. 2019;14:e0210578.
50. Wangdi K, Gatton ML, Kelly GC, Clements AC. Prevalence of asymptomatic malaria and bed net ownership and use in Bhutan, 2013: a country earmarked for malaria elimination. Malar J. 2014;13:352.

51. Nonaka D, Pongvongsa T, Nishimoto F, Nansounthavong P, Sato Y, Jiang $H$, et al. Households with insufficient bednets in a village with sufficient bednets: evaluation of household bednet coverage using bednet distribution index in Xepon District, Lao PDR. Trop Med Health. 2015:43:95-100.

52. Monroe A, Asamoah O, Lam Y, Koenker H, Psychas P, Lynch M, et al. Outdoor-sleeping and other night-time activities in northern Ghana: implications for residual transmission and malaria prevention. Malar J. 2015;14:35.

53. Koenker HM, Loll D, Rweyemamu D, Ali AS. A good night's sleep and the habit of net use: perceptions of risk and reasons for bed net use in Bukoba and Zanzibar. Malar J. 2013;12:203.

54. Von SL, Ikonomedis K, Mshamu S, Nkya TE, Mukaka M, Pell C, et al. Affordable house designs to improve health in rural Africa: a field study from northeastern Tanzania. Lancet Planet Health. 2017;1:e188-99.

55. Winch PJ, Makemba AM, Kamazima SR, Lwihula GK, Lubega P, Minjas $J \mathrm{~N}$, et al. Seasonal variation in the perceived risk of malaria: implications for the promotion of insecticide impregnated bed nets. Soc Sci Med. 1994;39:63-75.

56. Pattanasin S, Satitvipawee P, Wongklang W, Viwatwongkasem C, Bhumiratana A, Soontornpipit $P$, et al. Risk factors for malaria infection among rubber tappers living in a malaria control program area in southern Thailand, Southeast Asian. J Trop Med Public Health. 2012;43:1313-25.

57. Grietens KP, Xuan XN, Van BW, Duc TN, Ribera JM, Nhat TB, et al. Low perception of malaria risk among the Ra-glai ethnic minority in southcentral Vietnam: implications for forest malaria control. Malar J. 2010;9:23.

58. Lyttleton C. Deviance and resistance: malaria elimination in the greater Mekong subregion. Soc Sci Med. 2016;150:144-52.

59. Wharton-Smith A, Shafique MA. Qualitative study to assess consumer preferences and barriers to use of long lasting insecticidal nets in Myanmar. London: Malaria Consortium; 2014

60. Eisele TP, Bennett A, Simlumbe K, Finn TP, Porter TR, Chalwe V, et al. The impact of four rounds of mass drug administration with dihydroartemisinin-piperaquine in Southern Province, Zambia. Am J Trop Med Hyg 2020;103(Suppl 2):7-18.

61. WHO Malaria Policy Advisory Committee. Malaria Policy Advisory Committee to the WHO: conclusions and recommendations of eighth biannual meeting (September 2015). Malar J. 2016;15:117.

62. Govella NJ, Ferguson H. Why use of interventions targeting outdoor biting mosquitoes will be necessary to achieve malaria elimination. Front Physiol. 2012;3:199.

63. Smithuis FM, Kyaw MK, Phe UO, van der Broek I, Katterman N, Rogers $C$, et al. The effect of insecticide-treated bed nets on the incidence and prevalence of malaria in children in an area of unstable seasonal transmission in western Myanmar. Malar J. 2013;12:363.

64. Edwards HM, Sriwichai P, Kirabittir K, Prachumsri J, Chavez IF, Hii J. Transmission risk beyond the village: entomological and human factors contributing to residual malaria transmission in an area approaching malaria elimination on the Thailand-Myanmar border. Malar J. 2019;18:221.

65. Dondorp AM, Yeung S, White L, Nguon C, Day NP, Socheat D, et al. Artemisinin resistance: current status and scenarios for containment. Nat Rev Microbiol. 2010;8:272.

66. Hii J, Rueda LM. Malaria vectors in the Greater Mekong Subregion: overview of malaria vectors and remaining challenges. SE Asian J Trop Med. 2013:44:73-165.

67. Gryseels C, Durnez L, Gerrets R, Uk S, Suon S, Set S, et al. Re-imagining malaria: heterogeneity of human and mosquito behaviour in relation to residual malaria transmission in Cambodia. Malar J. 2015;14:165.

68. Hosking A. Efficacy of insecticide treated nets in South East Asia. In: Meek S, Kaviratne M, Eds. Malaria Consortium annotated bibliography; 2010. p.1-44.

69. Kolaczinski J, Macdonald M, Meek S. Vector control to eliminate artemisinin resistant malaria in the Greater Mekong Subregion. Lancet Infect Dis. 2014;14:9-11. 
70. Dutta P, Khan SA, Khan AM, Borah J, Sarmah CK, Mahanta J. The effect of insecticide-treated mosquito nets (ITMNs) on Japanese encephalitis virus seroconversion in pigs and humans. Am J Trop Med Hyg. 2011;84:466-72.

71. Mondal D, Huda MM, Karmoker MK, Ghosh D, Matlashewski G, Nabi SG,

et al. Reducing visceral leishmaniasis by insecticide impregnation of bednets, Bangladesh. Emerg Infect Dis. 2013;19:1131-4.

72. Google. (n.d.). [Tha Song Yang district, Tak province, Thailand]. https://is gd/8Vutps. Accessed 8 May 2020.
73. NordNordWest. Locator map of Tak Province, Thailand. https://tl.wikip edia.org/wiki/Talaksan:Thailand_Tak_locator_map.svg. Accessed 5 May 2020.

\section{Publisher's Note}

Springer Nature remains neutral with regard to jurisdictional claims in published maps and institutional affiliations.
Ready to submit your research? Choose BMC and benefit from:

- fast, convenient online submission

- thorough peer review by experienced researchers in your field

- rapid publication on acceptance

- support for research data, including large and complex data types

- gold Open Access which fosters wider collaboration and increased citations

- maximum visibility for your research: over $100 \mathrm{M}$ website views per year

At BMC, research is always in progress.

Learn more biomedcentral.com/submissions 